\title{
Short-Term Deprivation Does Not Influence Monocular or Dichoptic Temporal Synchrony at Low Temporal Frequency
}

Yiya Chen ${ }^{1 t}$, Seung Hyun Min't, Ziyun Cheng', Shijia Chen', Zili Wang', Chunwen Tao', Fan Lu', Jia Qu'*, Pi-Chun Huang ${ }^{3}$, Robert F. Hess² and Jiawei Zhou' ${ }^{1 *}$

${ }^{1}$ State Key Laboratory of Ophthalmology, Optometry and Vision Science, School of Ophthalmology and Optometry and Eye hospital, Wenzhou Medical University, Wenzhou, China, ${ }^{2}$ Department of Ophthalmology and Visual Sciences, McGill Vision Research, McGill University, Montreal, QC, Canada, ${ }^{3}$ Department of Psychology, National Cheng Kung University, Tainan, Taiwan

OPEN ACCESS

Edited by: Irene Senna,

Ulm University, Germany

Reviewed by:

Paola Binda,

University of Pisa, Italy

Donald Edward Mitchell,

Dalhousie University, Canada

*Correspondence:

Jia Qu

jqu@wz.zj.cn

Jiawei Zhou

zhoujw@mail.eye.ac.cn

these authors have contributed equally to this work

Specialty section: This article was submitted to

Perception Science,

a section of the journal

Frontiers in Neuroscience

Received: 23 December 2019

Accepted: 02 April 2020

Published: 28 April 2020

Citation:

Chen Y, Min SH, Cheng Z, Chen S, Wang Z, Tao C, Lu F, Qu J,

Huang P-C, Hess RF and Zhou J (2020) Short-Term Deprivation Does Not Influence Monocular or Dichoptic Temporal Synchrony at Low Temporal Frequency. Front. Neurosci. 14:402. doi: 10.3389/fnins.2020.00402
Studies on binocular combination and rivalry show that short-term deprivation strengthens the contribution of the deprived eye in binocular vision. However, whether short-term monocular deprivation affects temporal processing per se is not clear. To address this issue, we conducted a study to investigate the effect of monocular deprivation on dichoptic temporal synchrony. We tested ten adults with normal vision and patched their dominant eye with an opaque patch for $2.5 \mathrm{~h}$. A temporal synchrony paradigm was used to measure if temporal synchrony thresholds change as a result of monocular pattern deprivation. In this paradigm, we displayed two pairs of Gaussian blobs flickering at $1 \mathrm{~Hz}$ with either the same or different phased-temporal modulation. In Experiment 1, we obtained the thresholds for detecting temporal asynchrony under dichoptic viewing configurations. We compared the thresholds for temporal synchrony between before and after monocular deprivation and found no significant changes of the interocular synchrony. In Experiment 2, we measured the monocular thresholds for detecting temporal asynchrony. We also found no significant changes of the monocular synchrony of either the patched eye or the unpatched eye. Our findings suggest that short-term monocular deprivation induced-plasticity does not influence monocular or dichoptic temporal synchrony at low temporal frequency.

Keywords: monocular deprivation, temporal synchrony, interocular suppression, temporal processing, visual plasticity

\section{INTRODUCTION}

Hubel and Wiesel (1963) first demonstrated that visual experience in early life can shift ocular dominance in the feline visual system. For instance, the closure of one eye during the critical period, and hence blocking any form of visual input entering the eye, for a period of days or weeks shifts the eye dominance favoring the non-deprived eye. Monocular deprivation modifies ocular dominance in favor of the non-deprived eye at the expense of the 
deprived eye. This change was demonstrated both at the functional and structural levels of the ocular dominance columns in V1. They replicated the study in older cats and showed that the adult visual system is not as susceptible to visual experience (Hubel and Wiesel, 1970). This work ushered the belief that neural plasticity peaks immediately after birth and tapers off after the critical period.

Although monocular deprivation can shift the ocular dominance in favor of the non-deprived eye in young animals recent studies of humans have demonstrated that the adult visual system retains some degrees of neural plasticity, albeit of a different form (Levi, 2005; Thompson et al., 2008; Levi and Li, 2009; Lunghi et al., 2011; Clavagnier et al., 2013; Zhou et al., 2013a; Campana et al., 2014). For instance, patching an eye for a brief period of time (from $15 \mathrm{~min}$ to $5 \mathrm{~h}$ ) has been found to shift perceptual ocular dominance in adults favoring the deprived eye for only up to 30-90 min (Lunghi et al., 2011; Zhou et al., 2013a; Kim et al., 2017; Min et al., 2018). The shift in perceptual ocular dominance seems to be reciprocal, whereby the deprived eye's contribution to binocular vision strengthens and that of the non-deprived eye weakens. This reciprocal change in perceptual ocular dominance has been demonstrated with psychophysical methods, such as binocular rivalry and combination (Lunghi et al., 2011; Zhou et al., 2013a), for review see Basgoze et al. (2018). Zhou et al. (2014) and Kim et al. (2017) also showed that this deprivation effect could be induced without completely removing visual input in the deprived eye. Moreover, electrophysiological (Lunghi et al., 2015a; Zhou et al., 2015) and neuroimaging studies (Lunghi et al., 2015b; Chadnova et al., 2017; Binda et al., 2018) have also shown the reciprocal shift. To illustrate, Chadnova et al. (2017) reported an increased response of the deprived eye and decreased response of the non-deprived eye after short-term patching using MEG. They postulated that contralateral inhibition - which is known to regulate the contrast gain of each eye prior to binocular combination in a current model of binocular interaction (Meese et al., 2008) - mediates the patching effect (Chadnova et al., 2017). Using electrophysiology, Lunghi et al. (2015a) found that the amplitude of visually evoked potentials in the deprived eye increased whereas those from the non-deprived eye decreased. The strengthening of the deprived eye after short-term patching has been linked to reduced levels of GABA in the primary visual cortex (Lunghi et al., 2015b).

Short-term deprivation in human adults have also been shown to influence monocular visual functions. For example, Zhou et al. $(2013 \mathrm{a}, \mathrm{c})$ reported that short-term patching increases the contrast sensitivity of the deprived eye and decreases that of the non-deprived eye in both normal and amblyopic observers. Furthermore, Zhou et al. (2017) reported that the changes in monocular contrast sensitivity for chromatically defined stimuli were similar with that for achromatic- defined stimuli after 2.5h monocular deprivation. Finally, Binda et al. (2018), using fMRI BOLD responses, showed that monocular deprivation can affect selectivity for spatial frequency in V1. They found that the selectivity for high spatial frequencies was enhanced in the previously deprived eye. Although short-term monocular deprivation affects monocular visual functions, the reciprocal nature of these monocular changes suggest that the patching effect is based on binocular interaction.

Most psychophysical studies have measured the patching effect in the context of spatial vision with behavioral measurements such as binocular combination and rivalry. In a phase combination task, fusible horizontal gratings are shown to different eyes. Measurement of the bias in a fused percept can quantify each eye's contribution to binocular vision (Ding and Sperling, 2006; Zhou et al., 2013a). Conversely, two incompatible but orthogonal gratings are shown to different eyes in a binocular rivalry task. By measuring the perceived relative duration of each eye's grating stimulus for each subject, one can quantify the changes in eye dominance after patching (Lunghi et al., 2011). However, different neural mechanisms may be involved in these two psychophysical tasks (Bai et al., 2017; Baldwin and Hess, 2018). For testing monocular functions, gratings with different spatial frequency and different contrast are used to measure the contrast threshold (Zhou et al., 2013a). Although these psychophysical studies have demonstrated the spatial influence of short-term monocular visual deprivation in human adults, they have not shown whether patching influences temporal processing.

One aspect of temporal processing of visual information in the human relates to when an observer determines whether two stimuli are temporally synchronous. Temporal synchrony reflects the dynamic nature of visual processing. It has been shown to be an effective cue for binding and segmenting different signals in the absence of spatial cues (Rideaux et al., 2016). Temporal synchrony threshold, the minimum degree of temporal phase difference that enables observers to determine whether the target is flickering asynchronously in time, has been measured in the normal population (Hess and Maehara, 2011). It has also been used to assess temporal deficits in patients with amblyopia (Huang et al., 2012). Huang et al. (2012) reported that the temporal synchrony threshold of the amblyopic eye is higher than that of the fellow eye. They proposed that temporal processing deficit, rather than the detectability of the target, increases the temporal synchrony threshold in the amblyopic eye. Moreover, Tao et al. (2019) found that the elevation of temporal synchrony threshold in amblyopia was present not only when stimuli was presented to the amblyopic eye (i.e., monocular temporal synchrony) but also when presented dichoptically to amblyopic and fellow eyes (i.e., dichoptic temporal synchrony; or, interocular temporal delay). These findings suggest that there is clinical relevance to studying temporal processing in the human visual system.

In this study, we investigated whether short-term monocular deprivation could influence temporal processing of visual information, namely, the threshold for detecting temporal synchrony. A similar temporal synchrony paradigm was used as the one in the study of Tao et al. (2019). The patching effect was quantified by comparing the threshold for temporal synchrony before and after $2.5 \mathrm{~h}$ of monocular opaque patching. Specifically, thresholds for detecting dichoptic temporal asynchrony under dichoptic and monocular viewing were measured. Our results show that monocular deprivation does not influence either monocular or dichoptic temporal synchrony. 


\section{MATERIALS AND METHODS}

\section{Participants}

Ten subjects ( $23 \pm 0.42$ years old; four males) with normal or corrected-to-normal vision $(\log M A R \leq 0.0)$ participated in this study. All subjects were naive to the purpose of the study.

\section{Apparatus}

We performed our experiments with a Macintosh laptop equipped with Matlab (Mathworks, Natick, MA, United States) and the Psychtoolbox 3.0.14. We dichoptically displayed the stimuli on gamma-corrected head-mounted 3D goggles (Goovis Pro, NED Optics, Shenzhen, China). The OLED goggles had a resolution of $1600 \times 900$ pixels (corresponding to $46 \times 26$ degrees) and a refresh rate of $60 \mathrm{~Hz}$ in each eye. The maximal luminance of the OLED goggles was $150 \mathrm{~cd} / \mathrm{m}^{2}$.

The temporal response functions (TRFs) of the OLED monitor and Cathode Ray Tube (CRT) monitor are not the same. To address whether the TRF of the used display would confound our experimental results, we used Ito et al. (2013)'s measures of the TRFs for the CRT and OLED monitor (see Supplementary Figures S1 A,B in the supplementary) to simulate the display outputs and investigate whether the asynchrony signal in our test (i.e., the temporal lag) was varied across different TRFs. Two temporal profiles with a flickering rate of $1 \mathrm{~Hz}$ and $100 \mathrm{~ms}$ temporal lag were used in this simulation (Supplementary Figure S1C). The temporal profiles of the stimuli (Supplementary Figure S1C) were convolved with the TRFs for the CRT and OLED monitor (Supplementary Figures S1A,B) and the results showed the temporal lag did not change with the tested TRF (Supplementary Figures S1DF). In summary, the temporal lag threshold measured from our experiment did not confound the TRF of the OLED display. In fact, since our psychophysical task relied on the comparison between two dots, it would work on all the dots simultaneously regardless of which screen was used; it would not selectively affect one dot or dots in one eye. Thus, our measure of synchrony would not be limited by the screen response characteristics.

\section{Design}

All subjects participated in two experiments. Each experiment had three stages (Figure 1): baseline measurement of temporal synchrony before deprivation, monocular deprivation for $2.5 \mathrm{~h}$ and measurement of temporal synchrony after deprivation. We deprived the dominant eye [tested by the hole-in-the-card test (Dane and Dane, 2004)] for all subjects with an opaque patch (no transmission contrast or luminance). During patching, the participants performed typical office tasks such as browsing a web or reading.

We used a similar paradigm to Tao et al. (2019)'s study to measure the threshold for detecting temporal asynchrony. In this paradigm, two pairs of Gaussian blobs were presented; one pair flickered synchronously (i.e., reference), and the other asynchronously (i.e., signal). They flickered at a temporal frequency of $1 \mathrm{~Hz}$. The reason why we measured at this low temporal frequency was that a higher temporal frequency of the blobs would reduce precise measurement and this also ensured there were no afterimages. For Gaussian blobs with a temporal frequency of $1 \mathrm{~Hz}$ (the contrast of blob is modulated sinusoidal over time), one cycle of the stimuli included 60 frames in $1 \mathrm{~s}$ since our display screen had a refresh rate of $60 \mathrm{~Hz}$. Therefore, in this case, the minimum measurement accuracy would be 6 degrees (i.e., 360 degrees / 60 frames). On the other hand, at a higher temporal frequency, one cycle of the stimuli would include less than 60 frames, resulting in poorer measurement accuracy. In addition, we performed a pilot study using a higher temporal frequency of the blobs and found that the observers could not perform the task.

We modulated the temporal phase difference between the asynchronously flickering blobs to manipulate the degree of the asynchrony. The two blobs in each pair were presented diagonally with a separation of 2.46 degrees horizontally and vertically. The center of the two blobs was 4.3 degrees above or below the fixation. Between trials, the standard deviation of each Gaussian blob's size randomly varied from 0.28 to 0.46 degrees, and their luminance contrast varied from 0.4 to 0.8 to prevent participants from using local size or contrast cues to solve the task.

In Experiment 1, we measured the dichoptic temporal synchrony threshold in dichoptic viewing configuration ("Di" as shown in Figure 2A), in which both the signal and reference blobs were presented dichoptically to different eyes. In Experiment 2 , we measured the monocular temporal synchrony threshold in two additional monocular viewing configurations, i.e., $\mathrm{MD}$ (Figure 3A): monocular dominant eye (i.e., the assigned patched eye) viewing, where both signal and reference blobs were presented to the dominant eye; MND (Figure 3B): monocular non-dominant eye viewing, where both signal and reference blobs were presented to the non-dominant eye. Throughout this paper, we will refer to the three conditions with the abbreviations $\mathrm{Di}, \mathrm{MD}$, and MND.

All subjects performed each viewing configuration on a separate day. For each viewing configuration, the temporal synchrony threshold was measured before deprivation and at 0 , 10,20 , and $30 \mathrm{~min}$ after the $2.5-\mathrm{h}$ of monocular deprivation. An illustration of the experimental procedure is provided in Figure 1. Each test session contained 160 trials (eight temporal phase difference $\times 20$ repetitions) in one measure, which took about $5 \mathrm{~min}$ to complete. Before each experiment, subjects were asked to perform at least 160 practice trials.

\section{Procedure}

A constant stimuli method was used to measure the minimum degree of asynchrony that observers needed to discriminate the signal blobs (i.e., the pair of asynchronous blobs). Eight levels of temporal lag (i.e., temporal phase difference between the pair of asynchronous blobs), ranging from 33.33 to $266.67 \mathrm{~ms}$ and a step size of $33.33 \mathrm{~ms}$, were tested for each viewing configuration (i.e., $\mathrm{Di}, \mathrm{MD}$, and $\mathrm{MND}$ ). In each trial, the stimuli were presented for $1 \mathrm{~s}$. Participants were asked to determine whether the position of signal blobs was above or 


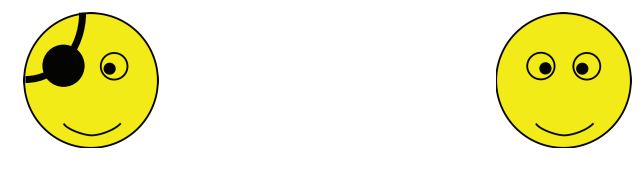

Baseline measurement
Patching (150 min)

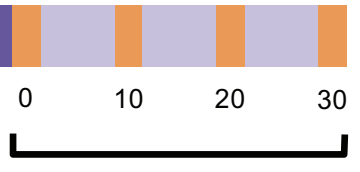

Post-patching measure
Testing

Patching

Normal Viewing

FIGURE 1 | An illustration of the experimental procedure. We deprived one eye for $2.5 \mathrm{~h}$, and assessed the temporal synchrony thresholds at baseline, and 0 , 10 , 20,30 min after the finish of the 2.5 -h of deprivation.

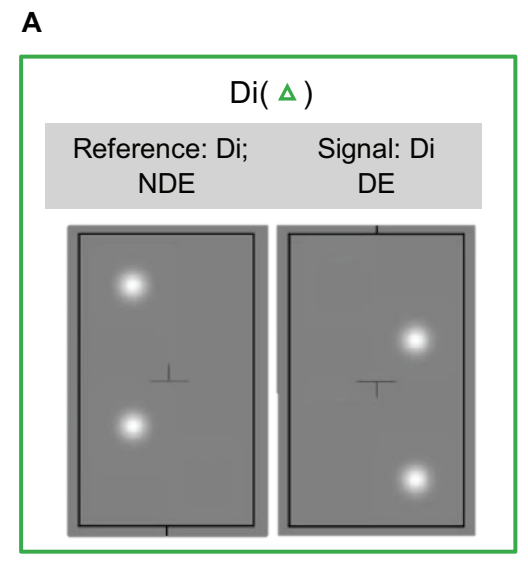

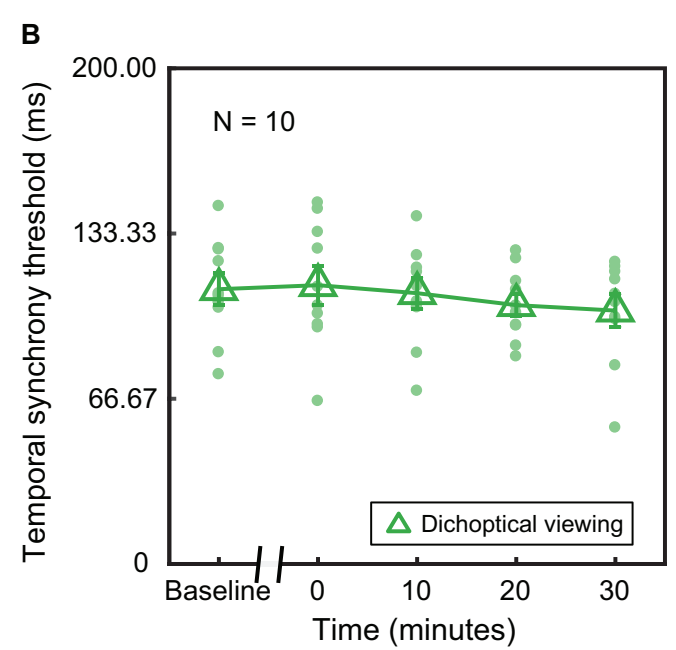

FIGURE 2 | Deprivation effect under dichoptic viewing configuration. (A) An illustration of the Di configuration. Both the signal and reference blobs were presented dichoptically to different eyes. (B) Dichoptic temporal synchrony threshold in the function of various time points before and after deprivation. Each green dot represents the threshold of each subject. Green triangle denotes the average threshold across ten subjects. Error bars represent standard errors.

below the fixation (two-alternative forced choice, 2AFC). The next trial started $750 \mathrm{~ms}$ after the participants' response. The eight levels of temporal lag were tested using an order randomized in various trials.

\section{Data Analysis}

For each participant, we derived the psychometric function defined as the proportion correct as a function of the temporal lag. The psychometric function of each configuration at each time point was fitted using Palamedes 1.8.1 (Prins and Kingdom, 2018) based on the following equation:

$$
\begin{array}{r}
\psi(x ; \alpha, \beta, \gamma, \lambda)=\gamma+(1-\gamma-\lambda) F(x ; \alpha, \beta) \\
=\gamma+(1-\gamma-\lambda)\left[1-\exp \left(-(x / \alpha)^{\beta}\right)\right]
\end{array}
$$

where, $\mathrm{F}(\mathrm{x} ; \alpha, \beta)$ is the Weibull function; $\mathrm{x}$ is the temporal lag; $\alpha$ is the threshold; $\beta$ is a free parameter related to the slope of the function; $\gamma$ is the guessed rate; and $\lambda$ is the lapse rate. During our fitting, we set $\gamma$ at 0.5 and constrained the $\lambda$ to a fixed value (ranging from 0 to 0.06 ) for each fitting. A maximum likelihood method was used for deriving the threshold and slope of the psychometric function for each testing time point of each subject.

\section{RESULTS}

\section{Experiment 1: Deprivation Effect Under Dichoptic Viewing Configuration (Di)}

To assess whether monocular deprivation influences dichoptic temporal synchrony (i.e., the minimum detectable interocular delay), we performed the Di configuration (Figure 2A). The averaged and individual temporal synchrony thresholds as a function of time before and after patching are plotted in Figure 2B. We conducted a Shapiro-Wilks test to check for normality assumption $(p>0.05)$. Then, a one-way repeatedmeasures ANOVA was used (one within-subject: time of measurements before and after patching) to check whether the changes in the temporal synchrony threshold induced by monocular deprivation was significantly different relative to the one measured in baseline. One-way repeated-measures 
A

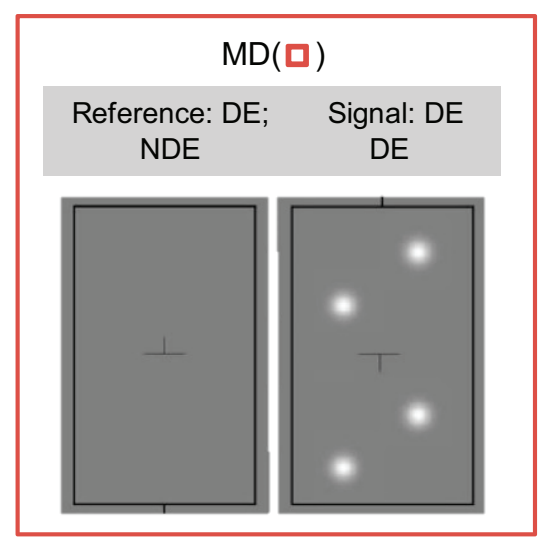

C

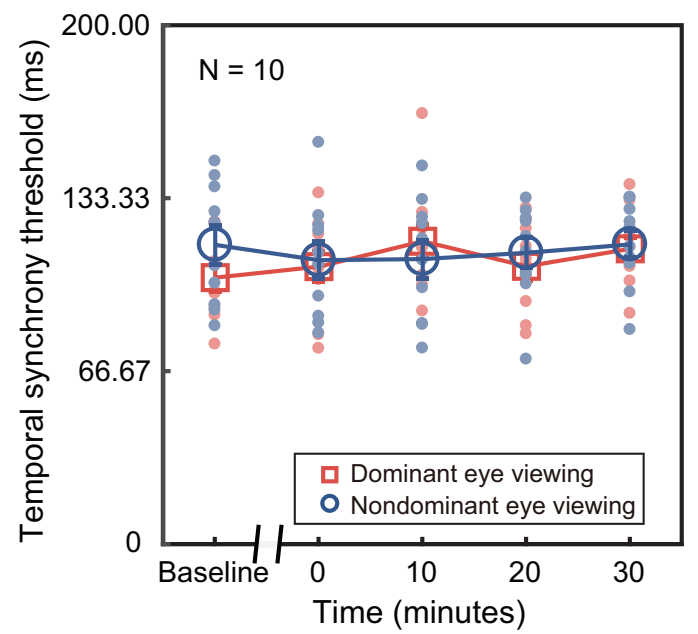

B

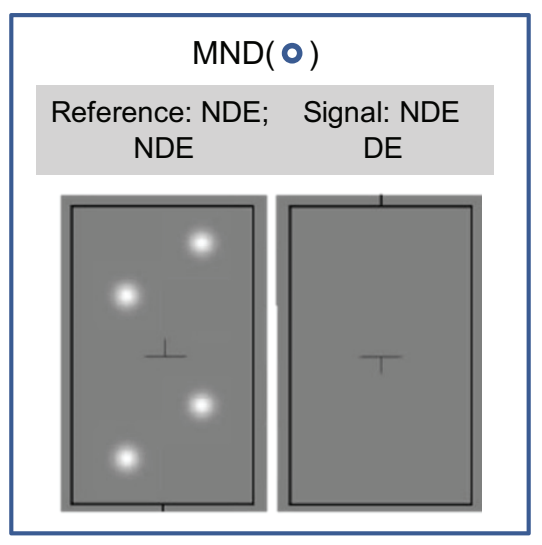

D

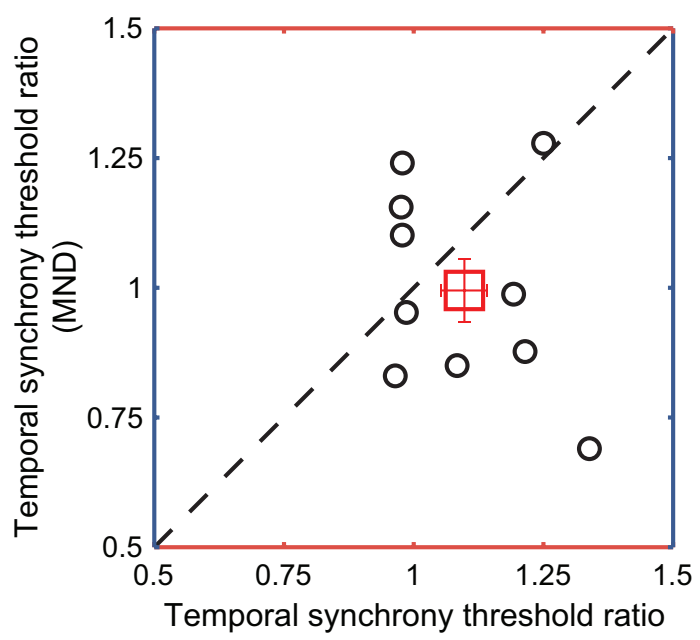

(MD)

FIGURE 3 | Deprivation effect under monocular viewing configurations. (A) Monocular dominant eye viewing (MD). Both signal and reference blobs were presented to the dominant eye (i.e., the assigned patched eye). (B) Monocular non-dominant eye viewing (MND). Both signal and reference blobs were presented to the non-dominant eye (i.e., the assigned unpatched eye). (C) Monocular temporal synchrony threshold in the function of various time points before and after deprivation. The red plot corresponds to MD configurations, and blue plot to MND. Each dot (blue or red) represents the threshold of each subject. Open symbols (blue circle or red square) denote the average threshold across ten subjects. Error bars (blue or red) represent standard errors. (D) Correlation between the changes of monocular temporal synchrony in the deprived eye and non-deprived eye. Error bars represent standard error.

ANOVA showed that the effect of deprivation on the temporal synchrony threshold was not significant $[F(4,36)=1.464$, $p=0.233]$. In other words, no significant difference in the dichoptic temporal synchrony threshold was found between before and after patching.

\section{Experiment 2: Deprivation Effect Under Monocular Viewing Configurations (MD and MND)}

To assess whether monocular deprivation shifts the threshold for temporal synchrony of one eye - be it the dominant (i.e., the assigned patched eye) or non-dominant eye - we obtained temporal synchrony thresholds in both the MD and MND configurations. The averaged and individual temporal synchrony thresholds as a function of time before and after patching are plotted in Figure 3C. One-way repeatedmeasures ANOVA showed that the effect of deprivation on the temporal synchrony threshold was not significant under either MD [i.e., the assigned patched eye: $F(4,36)=0.332$, $p=0.855$ ] or $\mathrm{MND}$ viewing configuration [i.e., the assigned unpatched eye: $F(4,36)=2.260, p=0.167]$. In short, we found no significant difference in the monocular temporal synchrony threshold before and after patching in both MD and MND configurations.

To further address whether there would be a difference in monocular temporal synchrony threshold between the deprived eye and the non-deprived eye, we conducted an additional twoway repeated-measures ANOVA, with the configuration (two levels) and time point of measurements after patching (four 
levels) selected as within-subject factors. We found that there was no significant difference between configurations $[F(1,9)=1.426$, $p=0.263]$ and time points $[F(3,27)=0.741, p=0.537]$. To better illustrate the relation between the changes of temporal synchrony in deprived eye and non-deprived eye, we divided the value of post-patching tests by the value of baseline to obtain the threshold ratio, and averaged the four post-test ratios. Then we plotted the averaged ratios of non-deprived eye as a function of the average ratios of the deprived eye in Figure 3D. There was no significant difference [paired- $t$ test, $t(9)=1.194 ; p=0.263$ ] and no significant correlation $(r=-0.318, p=0.370)$ between them.

\section{DISCUSSION}

In this study, we investigated whether short-term monocular deprivation could influence temporal processing between the two eyes using a temporal synchrony paradigm. Our results show that short-term monocular deprivation does not affect the dichoptic temporal synchrony threshold in normal observers.

Previous studies - be they psychophysical, neurophysiological or neuroimaging investigations - have reported that shortterm monocular deprivation induces neuroplastic changes in the visual system by shifting the perceptual ocular dominance in favor of the deprived eye (Lunghi et al., 2011; Zhou et al., 2013a). Both translucent (20\% luminance reduction) and opaque patches (no light transmission or contrast) have been shown to replicate the patching effect (Zhou et al., 2013a). A contrast-gain control model (Ding and Sperling, 2006) has been proposed to underlie the sensory balance between the eyes (Zhou et al., 2013a,b): During patching, the patched eye's contrast-gain would be elevated as a consequence of the loss of visual input. Immediately after patch removal, the previously deprived eye would have its contrast-gain restored to baseline values. Due to the reciprocal nature of the interocular inhibitory circuit (Meese et al., 2006), a reciprocal change would occur for the contrast gain of the non-deprived eye. This explanation is supported by both psychophysical (Zhou et al., 2013a) and neurophysiological studies (Lunghi et al., 2015a,b; Zhou et al., 2015; Chadnova et al., 2017). Assuming that changes in contrast-gain control result in changes in the speed of visual processing, we hypothesized that there may be reciprocal changes in the speed of visual processing in the two eyes after a period of monocular deprivation which might translate to elevated thresholds for temporal synchrony. However, our results show that no significant difference exists between the temporal synchrony thresholds before and after patching when stimuli are either dichoptically or monocularly presented.

Temporal synchrony provides an effective cue for integration and segmentation (Rideaux et al., 2016). Segmentation from temporal synchrony has been shown to be achieved by neurons in the early stage of visual processing (Goodbourn and Forte, 2013). An attenuated and delayed hemodynamic response function in early visual cortex (i.e., reduced synchrony of neural firing) due to abnormal interocular suppression, has been proposed as a possible cause for the temporal synchrony deficits in amblyopia (Farivar et al., 2011; Huang et al., 2012; Tao et al., 2019). Therefore, the processing of temporal synchrony occurs primarily in the early visual cortex. Moreover, electrophysiological studies (Lunghi et al., 2015a; Zhou et al., 2015) have suggested that short-term monocular patching can affect early visual areas, especially primary visual cortex (V1). Also Binda et al. (2018) confirmed that the effect of short-term monocular deprivation was most robust in $\mathrm{V} 1$, and moderate in V2, V3 and V4 but absent in V3a and hMT+ via fMRI. However, these studies mainly report the changes of response amplitude after patching rather than those of response timing. An unperturbed temporal synchrony threshold may be the result of little to no influence on the synchrony of neural firing by patching. However, there are multiple functional columns in V1 (Daw, 2006). Therefore, despite our findings of no effect on temporal synchrony detection from patching, it would be inappropriate to conclude that patching minimally affects temporal processing.

Another possible factor is that the patching effect on the temporal processing is too small to be detected by our paradigm. Hess and Maehara (2011) reported that we are surprisingly poor at making temporal synchrony judgements, of the order of 30 milliseconds. Therefore, we performed a power analysis based on the variance from our samples $(n=10)$, i.e., $\sigma_{d}=13.414$ for Di configuration, $\sigma_{d}=22.054$ for MD configuration, $\sigma_{d}=12.835$ for MND configuration. To reach a power of $80 \%$, the effect size would need to be $E=11.88 \mathrm{~ms}, E=19.53 \mathrm{~ms}$, and $E=11.36 \mathrm{~ms}$ for $\mathrm{Di}, \mathrm{MD}, \mathrm{MND}$ configuration, respectively. Thus, any change in temporal processing that occurs at a finer level than this and that impacts other temporal processes would not have been reflected in our approach using temporal synchrony.

Psychophysical studies on short-term monocular deprivation have shown conflicting results. It seems that findings from one task might not agree with those from other tasks because a specific psychophysical task can target distinct level of spatial processing for visual information. Binocular rivalry and combination tasks have shown different results from identical manipulation of visual information. For example, scrambling the phase of a dichoptic movie in one eye has been shown to elicit the patching effect in a binocular rivalry task (Bai et al., 2017) but not in a phase combination task (Zhou et al., 2014). Also, after short-period patching with a translucent patch, the changes in eye dominance were found to be much stronger and longerlasting for chromatically defined stimuli than achromatically defined ones in binocular rivalry (Lunghi et al., 2013), whereas the changes were similar for the two kinds of stimuli in binocular combination (Zhou et al., 2017). Baldwin and Hess (2018) used two different masks (parallel vs. cross-oriented) to mimic binocular rivalry and combination. Not finding any correlation between the decrease in detection threshold across the two masks, they concluded that short-term monocular deprivation induces multiple separable effects. We suspect that the task-difference of the patching effect that has been reported in the field of spatial vision might also exist in the temporal vision. Here we tested one specific example of temporal processing, namely temporal synchrony. A future study should investigate whether shortterm monocular deprivation affects other aspects of temporal 
processing in the visual system such as single-event asynchrony judgments, unimodal (visual) or cross-modal (e.g., audio-visual).

\section{DATA AVAILABILITY STATEMENT}

All datasets generated for this study are included in the Supplementary Data Sheet S1.

\section{ETHICS STATEMENT}

The studies involving human participants were reviewed and approved by Ethics Committee of the Wenzhou Medical University. The participants provided their written informed consent to participate in this study.

\section{AUTHOR CONTRIBUTIONS}

YC, SM, FL, JQ, P-CH, RH, and JZ conceived the experiments. $\mathrm{YC}, \mathrm{ZC}, \mathrm{SC}, \mathrm{ZW}$, and CT performed the experiments. YC, SM, $\mathrm{ZC}$, and $\mathrm{JZ}$ analyzed the data and interpreted the data. YC, SM, $\mathrm{P}-\mathrm{CH}, \mathrm{RH}$, and $\mathrm{JZ}$ wrote the manuscript. All authors contributed to manuscript revision, read and approved the submitted version.

\section{REFERENCES}

Bai, J., Dong, X., He, S., and Bao, M. (2017). Monocular deprivation of Fourier phase information boosts the deprived eye's dominance during interocular competition but not interocular phase combination. Neuroscience 352, 122-130. doi: 10.1016/j.neuroscience.2017.03.053

Baldwin, A. S., and Hess, R. F. (2018). The mechanism of short-term monocular deprivation is not simple: separate effects on parallel and crossoriented dichoptic masking. Sci. Rep. 8:6191. doi: 10.1038/s41598-018-24 584-9

Basgoze, Z., Mackey, A. P., and Cooper, E. A. (2018). Plasticity and adaptation in adult binocular vision. Curr. Biol. 28, R1406-R1413. doi: 10.1016/j.cub.2018. 10.024

Binda, P., Kurzawski, J. W., Lunghi, C., Biagi, L., Tosetti, M., and Morrone, M. C. (2018). Response to short-term deprivation of the human adult visual cortex measured with 7T BOLD. eLife 7:e40014. doi: 10.7554/eLife.40014

Campana, G., Camilleri, R., Pavan, A., Veronese, A., and Lo, G. G. (2014). Improving visual functions in adult amblyopia with combined perceptual training and transcranial random noise stimulation (tRNS): a pilot study. Front. Psychol. 5:1402. doi: 10.3389/fpsyg.2014.01402

Chadnova, E., Reynaud, A., Clavagnier, S., and Hess, R. F. (2017). Short-term monocular occlusion produces changes in ocular dominance by a reciprocal modulation of interocular inhibition. Sci. Rep. 7:41747. doi: 10.1038/srep41747

Clavagnier, S., Thompson, B., and Hess, R. F. (2013). Long lasting effects of daily theta burst rTMS sessions in the human amblyopic cortex. Brain Stimul. 6, 860-867. doi: 10.1016/j.brs.2013.04.002

Dane, A., and Dane, S. (2004). Correlations among handedness, eyedness, monocular shifts from binocular focal point, and nonverbal intelligence in university mathematics students. Percept. Mot. Skills 99, 519-524. doi: 10.2466/ Pms.99.6.519-524

Daw, N. W. (2006). Visual Development. Basel: Springer, doi: 10.1007/0-38730484-3

Ding, J., and Sperling, G. (2006). A gain-control theory of binocular combination. Proc. Natl. Acad. Sci. U.S.A. 103, 1141-1146. doi: 10.1073/pnas.050962 9103

\section{FUNDING}

This work was supported by the National Natural Science Foundation of China Grants NSFC 31970975 and 81500754, the Qianjiang Talent Project (QJD1702021) and the Wenzhou Medical University grant QTJ16005 to JZ, the Canadian Institutes of Health Research Grants CCI-125686, NSERC grant 228103, and an ERA-NET Neuron grant (JTC2015) to RH. The sponsor or funding organization had no role in the design or conduct of this research.

\section{SUPPLEMENTARY MATERIAL}

The Supplementary Material for this article can be found online at: https://www.frontiersin.org/articles/10.3389/fnins. 2020.00402/full\#supplementary-material

FIGURE S1 | The temporal characteristics for the CRT and OLED monitor. (A,B) The temporal response function (TRF) for CRT and OLED. (C) The temporal profiles of the stimuli. The solid and dashed lines represent the two stimuli. Two stimuli flickered at $1 \mathrm{~Hz}$ and with $100 \mathrm{~ms}$ temporal lag. (D,E) The temporal profiles convolved with the TRF by using CRT and OLED. (F) Temporal lags shown in CRT and OLED. Black column denotes temporal lag we set for two stimuli; red one denotes temporal lag when stimuli appear on CRT display; green one denotes temporal lag when stimuli appear on OLED display.

DATA SHEET S1 | The datasets generated for this study.

Farivar, R., Thompson, B., Mansouri, B., and Hess, R. F. (2011). Interocular suppression in strabismic amblyopia results in an attenuated and delayed hemodynamic response function in early visual cortex. J. Vis. 11:16. doi: 10. $1167 / 11.14 .16$

Goodbourn, P. T., and Forte, J. D. (2013). Spatial limitations of fast temporal segmentation are best modeled by V1 receptive fields. J. Vis. 13:23. doi: 10.1167/ 13.13 .23

Hess, R. F., and Maehara, G. (2011). Does cognitive perception have access to brief temporal events? I-Perception 2, 142-149. doi: 10.1068/i0418

Huang, P. C., Li, J. R., Deng, D. M., Yu, M. B., and Hess, R. F. (2012). Temporal synchrony deficits in amblyopia. Invest. Ophthalmol. Vis. Sci. 53, 8325-8332. doi: $10.1167 /$ iovs.12-10835

Hubel, D. H., and Wiesel, T. N. (1963). Receptive fields of cells in striate cortex of very young, visually inexperienced kittens. J. Neurophysiol. 26, 994-1002. doi: 10.1152/jn.1963.26.6.994

Hubel, D. H., and Wiesel, T. N. (1970). The period of susceptibility to the physiological effects of unilateral eye closure in kittens. J. Physiol. 206, 419-436. doi: 10.1113/jphysiol.1970.sp009022

Ito, H., Ogawa, M., and Sunaga, S. (2013). Evaluation of an organic light-emitting diode display for precise visual stimulation. J. Vis. 13:6. doi: 10.1167/13.7.6

Kim, H. W., Kim, C. Y., and Blake, R. (2017). Monocular perceptual deprivation from interocular suppression temporarily imbalances ocular dominance. Curr. Biol. 27, 884-889. doi: 10.1016/j.cub.2017.01.063

Levi, D. M. (2005). Perceptual learning in adults with amblyopia: a reevaluation of critical periods in human vision. Dev. Psychobiol. 46, 222-232. doi: 10.1002/dev. 20050

Levi, D. M., and Li, R. W. (2009). Perceptual learning as a potential treatment for amblyopia: a mini-review. Vision Res. 49, 2535-2549. doi: 10.1016/j.visres.2009. 02.010

Lunghi, C., Berchicci, M., Morrone, M. C., and Di Russo, F. (2015a). Short-term monocular deprivation alters early components of visual evoked potentials. J. Physiol. 593, 4361-4372. doi: 10.1113/Jp270950

Lunghi, C., Burr, D. C., and Morrone, C. (2011). Brief periods of monocular deprivation disrupt ocular balance in human adult visual cortex. Curr. Biol. 21, R538-R539. doi: 10.1016/j.cub.2011.06.004 
Lunghi, C., Burr, D. C., and Morrone, M. C. (2013). Long-term effects of monocular deprivation revealed with binocular rivalry gratings modulated in luminance and in color. J. Vis. 13:1. doi: 10.1167/13.6.1

Lunghi, C., Emir, U. E., Morrone, M. C., and Bridge, H. (2015b). Short-term monocular deprivation alters GABA in the adult human visual cortex. Curr. Biol. 25, 1496-1501. doi: 10.1016/j.cub.2015.04.021

Meese, T. S., Challinor, K. L., and Summers, R. J. (2008). A common contrast pooling rule for suppression within and between the eyes. Vis. Neurosci. 25, 585-601. doi: 10.1017/S095252380808070x

Meese, T. S., Georgeson, M. A., and Baker, D. H. (2006). Binocular contrast vision at and above threshold. J. Vis. 6, 1224-1243. doi: 10.1167/6.11.7

Min, S. H., Baldwin, A. S., Reynaud, A., and Hess, R. F. (2018). The shift in ocular dominance from short-term monocular deprivation exhibits no dependence on duration of deprivation. Sci. Rep. 8:17083. doi: 10.1038/s41598-018-35084-1

Prins, N., and Kingdom, F. (2018). Applying the model-comparison approach to test specific research hypotheses in psychophysical research using the palamedes toolbox. Front. Psychol. 9:1250. doi: 10.3389/fpsyg.2018.01250

Rideaux, R., Badcock, D. R., Johnston, A., and Edwards, M. (2016). Temporal synchrony is an effective cue for grouping and segmentation in the absence of form cues. J. Vis. 16:23. doi: 10.1167/16.11.23

Tao, C. W., Wu, Y. D., Gong, L., Chen, S. J., Mao, Y., Chen, Y. Y., et al. (2019). Abnormal monocular and dichoptic temporal synchrony in adults with Amblyopia. Invest. Ophthalmol. Vis. Sci. 60, 4858-4864. doi: 10.1167/iovs.1927893

Thompson, B., Mansouri, B., Koski, L., and Hess, R. F. (2008). Brain plasticity in the adult: modulation of function in amblyopia with rTMS. Curr. Biol. 18, 1067-1071. doi: 10.1016/j.cub.2008.06.052

Zhou, J., Baker, D. H., Simard, M., Saint-Amour, D., and Hess, R. F. (2015). Short-term monocular patching boosts the patched eye's response in visual cortex. Restor. Neurol. Neurosci. 33, 381-387. doi: 10.3233/Rnn-14 0472

Zhou, J., Clavagnier, S., and Hess, R. F. (2013a). Short-term monocular deprivation strengthens the patched eye's contribution to binocular combination. J. Vis. 13:12. doi: $10.1167 / 13.5 .12$

Zhou, J., Jia, W., Huang, C. B., and Hess, R. F. (2013b). The effect of unilateral mean luminance on binocular combination in normal and amblyopic vision. Sci. Rep. 3:2012. doi: 10.1038/srep02012

Zhou, J., Reynaud, A., and Hess, R. F. (2014). Real-time modulation of perceptual eye dominance in humans. Proc. Biol. Sci. 281:20141717. doi: 10.1098/rspb. 2014.1717

Zhou, J., Reynaud, A., Kim, Y. J., Mullen, K. T., and Hess, R. F. (2017). Chromatic and achromatic monocular deprivation produce separable changes of eye dominance in adults. Proc. Biol. Sci. 284:20171669. doi: 10.1098/rspb.2017.1669

Zhou, J., Thompson, B., and Hess, R. F. (2013c). A new form of rapid binocular plasticity in adult with amblyopia. Sci. Rep. 3:2638. doi: 10.1038/srep0 2638

Conflict of Interest: The authors declare that the research was conducted in the absence of any commercial or financial relationships that could be construed as a potential conflict of interest.

Copyright (c) 2020 Chen, Min, Cheng, Chen, Wang, Tao, Lu, Qu, Huang, Hess and Zhou. This is an open-access article distributed under the terms of the Creative Commons Attribution License (CC BY). The use, distribution or reproduction in other forums is permitted, provided the original author(s) and the copyright owner(s) are credited and that the original publication in this journal is cited, in accordance with accepted academic practice. No use, distribution or reproduction is permitted which does not comply with these terms. 